\title{
Eliminating homelessness in Canada through housing first: from demonstration project to policy and program change
}

\author{
Geoffrey Nelson* \\ Department of Psychology, Wilfrid Laurier University, Waterloo, Ontario, Canada
}

\section{Introduction}

Homelessness is a complex social problem that encompasses personal, interpersonal, and community dimensions that occur in a socio-political context. Complex problems, like homelessness, are uncertain and constantly in flux [1]. and require complex community interventions to address them [2]. It has been estimated that in Canada, there are 35,000 people who are homeless on a given night, and at least 235,000 people who experience homelessness every year [3]. In this paper, I tell the story of how Canada is using the Housing First (HF) approach to eliminate homelessness.

Until recently, the homelessness sector in Canada has lacked a research base for evidence-based practice. This began to change in 2009 when the federal government funded and the Mental Health Commission of Canada administered a large-scale research demonstration project in homelessness. At Home/Chez Soi was a randomized controlled trial of HF that was implemented in five Canadian cities with over 2,000 participants with experiences of mental illness and homelessness enrolled in the study [4].

$\mathrm{HF}$ is a complex community intervention whose main components are rent supplements and a clinical intervention, typically Assertive Community Treatment (ACT) or Intensive Case Management (ICM) $[5,6]$. While evidence-based practices typically emphasize fidelity to a program model, complex community interventions must adapt to

different community contexts. An important distinction has been made regarding this tension between the form and function of the intervention [7]. A complex community intervention need not rigidly follow a specific format (i.e., form), but it must adhere to the underlying principles of the intervention (i.e., its functions). HF takes this distinction into account in the following way. HF is based on set of principles (i.e., consumer choice, separation of housing and treatment, community integration), but it can be adapted for different populations in different contexts. In At Home/Chez Soi, HF was adapted for Indigenous communities in Winnipeg to include culturally relevant practices (e.g., sweats, the incorporation of elders), and in Toronto it was adapted for ethnoracial populations to address racism and discrimination in the lived experience of participants.

The At Home/Chez Soi research showed that HF participants had outcomes that were superior to those receiving usual treatment (e.g., shelters, transitional housing). Over two years, HF participants achieved significantly greater housing stability, quality of life, and community functioning, and these outcomes were observed for participants receiving ACT or ICM $[8,9]$. These findings were consistent with the results of previous trials and quasi-experiments of HF conducted in the United States [10].
Given the positive findings from the At Home/Chez Soi trial of HF, the question for translational science is how to scale up and scale out HF across Canada. Scaling up refers to systems transformation to support a new way of serving people, while scaling out refers to efforts to diffuse and replicate an evidence-based practice into new communities or to expand its implementation in its current context [1]. Change efforts were undertaken to effect both scaling up and scaling out of HF.

With regard to scaling up, the Mental Health Commission and At Home/Chez Soi researchers, practitioners, and people with lived experience informed the federal government and, particularly, the Prime Minister's Office, about the effectiveness of HF. As a result of these efforts and other pressure, the federal government renewed and repurposed its Homelessness Partnering Strategy that provides funding to 61 Canadian communities to address homelessness. Specifically, the majority of funding was to be used to implement HF programs. This represented a dramatic shift in policy. Our qualitative research identified several factors that led to this change including the importance of evidence, strong relationships between researchers and decision-makers, and the expertise of key stakeholders [11].

Systems transformation was also evident at the community level [12]. Case studies of six communities striving to implement HF found changes in the capacity of the service delivery system to implement HF, changes in the coordination of parts of the service delivery system, and increased collaboration among local stakeholders to enhance HF implementation. These changes were facilitated or constrained by the larger context of evidence, climate, policy, and funding.

To promote scaling out of HF to new settings, the Mental Health Commission funded a three-year training and technical assistance program to help 18 communities interested in implementing HF. In our research, we conducted intensive case studies with six of these communities to understand how HF could be scaled out. The training and technical assistance included two on-site trainings (one early in the process and one later), trainings in four regions of Canada, regular telephone consultation, community of practice phone calls, on-site fidelity assessments, and the development of regional networks [1315]. We found that in the six communities, 14 new, high-fidelity HF programs were created [14]. Several factors facilitated implementation, including addressing misconceptions about HF, encouraging teambased practice, having a housing specialist, helping leaders connect

${ }^{\star}$ Correspondence to: Geoffrey Nelson, Department of Psychology, Wilfrid Laurier University, Waterloo, Ontario, Canada, Email: gnelson@wlu.ca

Received: November 02, 2018; Accepted: February 04, 2019; Published: February 07, 2019 
with others facing similar challenges, and addressing context specific implementation issues. Insufficient rent supplements and a lack of staff capacity impeded implementation. The policy context regarding the availability of rent supplements and use of ACT teams also impacted HF implementation.

Translating evidence into policy and practice is a complex undertaking that involves multiple stakeholders, systems, and competing perspectives and demands multiple strategies for change. In spite of many challenges, research in Canada has shown that HF can be effectively disseminated to policy-makers and new communities leading to changes in policy and practice. More broadly, HF is now being widely adopted elsewhere, including Europe, Australia, and New Zealand [11]. Nevertheless, it is important to take into account the ever-changing social-political context. Through its National Housing Strategy, the federal government of Canada has pledged its intentions to rectify the erosion of low-income, social housing. While eliminating homelessness continues to be a goal, the commitment to HF is no longer evident in the new policy [16]. Thus, new interventions are needed to keep Canada on course with HF as a key strategy for eliminating homelessness in Canada.

\section{Acknowledgements}

This research was funded by the Canadian Institutes of Health Research (CIHR), Partnerships for Health Systems Improvement (PHSI) program (grant number 293443), and St. Michael's Hospital of Toronto, and was supported by the Tri-Council Research Support Fund. Thanks to community partners, the Mental Health Commission of Canada, and research and decision-maker partners for their participation in this research. I dedicate this paper to Dr. Paula Goering, Co-Principal Investigator for this research, who passed away on May 24, 2016.

\section{References}

1. Moore M, Westley F (2011) Surmountable chasms: networks and social innovation for resilient systems. Ecol Soc 16: 5

2. Greenhalgh T, Papoutsi C (2018) Studying complexity in health services research: desperately seeking an overdue paradigm shift. BMC Med 16: 95.
3. Gaetz S, Dej E, Richter T, Redman M (2016) The state of homelessness in Canada. Toronto: Canadian Observatory on Homelessness Press.

4. Goering P, Streiner DL, Adair C, Aubry T, Barker J, et al. (2011) The At Home/Chez Soi trial protocol: a pragmatic, multi-site, randomised controlled trial of a Housing First intervention for homeless individuals with mental illness in five Canadian cities. BMJ Open PP: 1. [Crossref]

5. Tsemberis S (2015) Housing First: The Pathways model to end homelessness for people with mental illness and addiction. Hazelden, Center City, MN.

6. Nelson G, Macnaughton E, Goering P (2015) What qualitative research can contribute to a randomized controlled trial of a complex community intervention. Contemp Clin Trials 45: 377-384. [Crossref]

7. Hawe P, Shiell A, Riley D (2004) Complex interventions: how 'out of control' can a randomized controlled trial be? BMJ 328: 1561-1563. [Crossref]

8. Aubry T, Goering P, Veldhuizen S, Adair CE, Bourque J, et al. (2016) A multiple city RCT of Housing First with Assertive Community Treatment for homeless Canadians with serious mental illness. Psych Serv 67: 275-281. [Crossref]

9. Stergiopoulos V, Hwang SW, Gozdzik A, Nisenbaum R, Latimer E, et al. (2015) Effect of scattered-site housing using rent supplements and intensive case management on housing stability among homeless adults with mental illness: a randomized controlled trial. JAMA 313: 905-915.

10. Aubry T, Nelson G, Tsemberis S (2015) Pathways Housing First for people with severe mental illness who are homeless: a review of the research. Can J Psychiatry 60: 467474. [Crossref]

11. Macnaughton E, Nelson G, Goering P, Piat M (2017) Moving evidence into policy: the story of the At Home/Chez Soi initiative's impact on federal homelessness policy in Canada and its implications for the spread of Housing First in Europe and internationally. Eur J Homelessness 11: 109-130.

12. Nelson G, Worton SK, Macnaughton E, Tsemberis SJ (2018) Systems change in the context of an initiative to scale up Housing First in Canada. J Community Psychol.

13. Worton SK, Hasford J, Macnaughton E, Nelson G, MacLeod T, et al. (2018) Understanding system changes for early implementation of Housing First: an examination of facilitators/barriers, training/technical assistance, and points of leverage. Am J Community Psychol 61:118-130.

14. Macnaughton E, Nelson G, Worton SK, Tsemberis S, Stergiopoulos V, et al. (2018) Navigating complex implementation contexts: overcoming challenges and achieving outcomes in a national initiative to scale out Housing First in Canada. Am J Community Psychol.

15. Worton SK. Networking strategies to promote the implementation of Housing First in Canada.

16. Canada's National Housing Strategy, 2017.

Copyright: ( 2019 Nelson G. This is an open-access article distributed under the terms of the Creative Commons Attribution License, which permits unrestricted use, distribution, and reproduction in any medium, provided the original author and source are credited. 